'Российская медицинская академия непрерывного профессионального образования, Москва, Россия

${ }^{2}$ Медико-генетическая лаборатория ООО «Эвоген», Москва, Россия

${ }^{3}$ Московский клинический научно-практический центр имени А.С. Логинова, Москва, Россия

${ }^{4}$ Национальный медицинский исследовательский центр эндокринологии, Москва, Россия

${ }^{5}$ Российский университет дружбы народов, Москва, Россия

Около 5-10\% злокачественных новообразований (3НО) являются наследственными. Носители мутаций, ассоциированных с наследственными опухолевыми синдромами (HOC), подвержены высокому риску развития опухолей в детском и молодом возрасте, а также синхронных и метахронных множественных опухолей. При этом данная группа заболеваний остается в основном онкологической проблемой, а клинические решения принимаются только при выявлении $3 \mathrm{HO}$ у носителей патогенных мутаций.

Для носителей мутаций, ассоциированных с НОС, должны быть разработаны индивидуальные рекомендации по скринингу, лечению и профилактике онкологических заболеваний, чтобы предотвратить неблагоприятный исход заболевания. Важна идентификация пациентов группы риска врачами всех специальностей для дальнейшего направления на медико-генетическое консультирование с молекулярно-генетическим тестированием (при наличии показаний). Остаются открытыми вопросы стандартизации критериев отбора для проведения генетических тестов, дальнейшей тактики профилактики, скрининга и лечения большого количества наследственных онкологических заболеваний.

Этот обзор создан с целью информирования врачей разных специальностей, в том числе эндокринологов, о НОС. Он позволит ознакомиться с основными клиническими особенностями некоторых синдромов, облегчит понимание разницы между наследственным и ненаследственным раком, распознавание признаков наследственного рака, а также познакомит с показаниями для генетического обследования и генетического консультирования больного. Вместе с тем существенные различия между международными и отечественными рекомендациями по скрининговым мероприятиям, диагностике и лечению НОС определяют необходимость пересмотра существующих и разработки новых алгоритмов медицинского сопровождения пациентов с НОС.

КЛЮЧЕВЫЕ СЛОВА: наследственные опухолевые синдромы; моногенные заболевания; молекулярно-генетическая диагностика; генетическое консультирование; скрининг; полногеномное секвенирование.

\title{
HEREDITARY CANCER SYNDROMES: A MODERN PARADIGM
}

(c) Elena E. Baranova ${ }^{1,2 *}$, Natalia A. Bodunova ${ }^{3}$, Maria V. Vorontsova ${ }^{4}$, Galina S. Zakharova ${ }^{4}, M^{2}$ aria V. Makarova ${ }^{2,5}$, Pavel O. Rumyantsev ${ }^{4}$, Igor E. Hat'kov ${ }^{3}$

${ }^{1}$ Russian Medical Academy of Continuous Professional Education, Moscow, Russia

${ }^{2}$ LLC Evogen, Moscow, Russia

${ }^{3}$ The Loginov Moscow Clinical Scientific Center, Moscow, Russia

${ }^{4}$ Endocrinology Research Center, Moscow, Russia

${ }^{5}$ Russian People's Friendship University (RUDN University), Moscow, Russia

About $5-10 \%$ of malignant neoplasms (MN) are hereditary. Carriers of mutations associated with hereditary tumor syndromes (HTS) are at high risk of developing tumors in childhood and young age and synchronous and metachronous multiple tumors. At the same time, this group of diseases remains mainly an oncological problem, and clinical decisions are made only when MNs are detected in carriers of pathogenic mutations.

Individual recommendations for cancer screening, treatment, and prevention should be developed for carriers of mutations associated with HTS to prevent an adverse outcome of the disease. It is essential to identify patients at risk by doctors of all specialties for further referral to medical and genetic counseling with molecular genetic testing (in case of indications). The problems of standardization of enrollment criteria for genetic tests, further tactics of prevention, screening, and treatment of many hereditary oncological diseases remain unsolved.

This review was created to inform doctors of various specialties, including endocrinologists, about the HTS. This allows them to get acquainted with main clinical features of specific syndromes, helps to understand the difference between hereditary and non-hereditary cancer, recognize signs of hereditary cancer, and introduce the indications for genetic examination and genetic counseling of the patient. Also, significant differences between international and domestic recommendations 
on screening measures, diagnosis, and treatment of HTS underline the need to review the existing and develop new algorithms for medical support of patients with HTS.

KEYWORDS: hereditary cancer syndromes; single-gene defects; genetic testing; genetic counseling; genetic carrier screening; next generation sequencing.

Врачи практически любых специальностей на протяжении своей карьеры сталкиваются с наследственными опухолевыми синдромами (НОС). На наследственные формы рака приходится 5-10\% всех злокачественных новообразований (3НО) [1, 2]. Важно идентифицировать этих пациентов, поскольку им требуется особое долгосрочное медицинское сопровождение. Необходимым является наблюдение родственников, у которых может быть повышенный риск развития рака. Поэтому врач соответствующей специальности и врач-генетик должны подробно информировать пациентов и их кровнородственных членов семьи, обратившихся на консультацию, о генетически детерминированных рисках развития $3 \mathrm{HO}$ и о конкретных скрининговых обследованиях. Пациенты, у которых есть подозрение на НОС, всегда должны быть под наблюдением целого ряда специалистов, то есть им необходим широкий междисциплинарный подход.

\section{ПРИЧИНЫ НАСЛЕДСТВЕННЫХ ОПУХОЛЕВЫХ СИНДРОМОВ}

При НОС человек из-за наследуемой мутации имеет повышенный риск развития определенных опухолей, которые могут манифестировать в молодом или детском возрасте. Чаще всего опухоль возникает на 20-25 лет раньше, чем в случае спорадического рака той же локализации. Как правило, повышенный риск развития рака обусловлен мутацией одного гена (моногенные наследственные заболевания). Соответствующие гены обычно выполняют контрольные функции в регуляции клеточного цикла, восстановления повреждений ДНК или клеточной гибели. Опухоли, характерные для НОС, часто имеют определенные морфологические и иммуногистохимические особенности.

Спорадически возникающие (ненаследственные) опухоли могут быть вызваны мутациями в тех же генах, что и НОС, однако в этом случае генетические изменения в клетках соответствующей ткани развиваются в течение жизни конкретного пациента. В отличие от наследственных, соматические мутации (мутации в отдельных клетках организма, но не в половых) не передаются потомству.

Большинство наследственных онкологических заболеваний связаны с «мутацией зародышевой линии», герминальной мутацией, которая попадает в зиготу с яйцеклеткой или сперматозоидом и, следовательно, присутствует в каждой клетке нового организма. Таким образом, первый шаг в развитии рака уже сделан. Это объясняет, почему пациенты с НОС имеют чрезвычайно высокий риск развития заболевания и преимущественно в молодом возрасте. По одной из гипотез, это связано с 2-ступенчатой инактивацией гена. Злокачественная трансформация происходит тогда, когда в клетках организма возникают вовлеченные в онкологический процесс соматические мутации в том же гене, где и герминальная [3]. Вместе с тем в настоящее время существуют и другие объяснения патогенеза НОС [4]. Большинство НОС имеют аутосомно-доми- нантный тип наследования, при котором существует 50\% вероятность передачи патогенной мутации потомству.

Поскольку ЗНО широко распространены в популяции, необходимо сформировать показания для проведения скрининга НОС и молекулярно-генетического анализа.

\section{АНАМНЕЗ НАСЛЕДСТВЕННЫХ ОПУХОЛЕВЫХ СИНДРОМОВ}

Тщательный сбор анамнеза крайне важен для выявления пациентов с повышенным риском НОС.

Информация, требующаяся для клинико-генеалогического анализа, обязательно должна содержать данные о случаях рака у пробанда и у его кровных родственников (1 и 2 степени родства); о возрасте, в котором опухоли были диагностированы; данные о дополнительных факторах, повышающих риск НОC (например, принадлежность к ашкеназским евреям повышает вероятность наличия мутаций в BRCA1 и BRCA2).

Основные признаки НОС (особенно если отмечается одновременно несколько):

- семейный анамнез, обуславливающий высокий риск развития злокачественной опухоли у конкретного человека или членов семьи (кровных родственников), в частности, диагностированный у нескольких прямых родственников такой же тип рака (особенно в случае редких заболеваний), как у пациента, или различные типы рака у нескольких родственников;

- развитие нескольких типов опухолей (синхронно или метахронно);

- двустороннее поражение органов;

- необычно ранний возраст начала заболевания у пациента (например, колоректальный рак до 30 лет) или случаи детского рака у его сиблингов;

- предрасположенность к определенным типам опухолей в одной этнической группе;

- врожденные дефекты, ассоциированные с НОС (например, определенные пигментные пятна на коже и костные аномалии, ассоциированные с развитием нейрофиброматоза I типа);

- несвойственный для данного пола тип рака (например, рак молочной железы у мужчин).

Также по каждому типу рака существуют признаки, которые повышают вероятность наличия НОС [5].

В этих случаях целесообразно направить пациента и его родственников к врачу-генетику для более тщательного сбора анамнеза. При необходимости врач-генетик порекомендует дополнительные диагностические процедуры и предложит индивидуальные схемы скрининга для ранней диагностики. Скрининговые мероприятия могут облегчить раннюю диагностику рака и лечение предраковых состояний, тем самым улучшая прогноз.

Стоит отметить, что зачастую наличие в семье нескольких членов с опухолями одного типа связано не с генетическими факторами, а с общими для них вредными факторами среды или образом жизни (например, курение или 
ожирение). Поэтому при описании семейной истории необходимо оценить и наличие негенетических факторов риска.

\section{ДИАГНОСТИКА И СКРИНИНГ НАСЛЕДСТВЕННЫХ ОПУХОЛЕВЫХ СИНДРОМОВ}

Методы визуализации играют важную роль в скрининге и диагностике НОС. Многие опухоли, ассоциированные с НОС, имеют характерные черты [6, 7]. Если врач-радиолог знаком с данными признаками, он может заподозрить опухоль, связанную с НОС.

Для скрининга подобных опухолей используют различные типы визуализации. Для щитовидной железы, яичников, яичек и почек применяют ультразвуковую диагностику. Для молочных желез, мозга, шеи и брюшной полости - магнитно-резонансную томографию (МРТ) с контрастированием. Также применяется МРТ всего тела, например, для пациентов с мутациями ТP53 [8]. В работе Anupindi S.A. и соавт. продемонстрировано, что чувствительность и специфичность такой МРТ достигает 100 и 94\% соответственно для детей с НОС [9]. В настоящее время проводится клиническое МРТ-тестирование всего тела для раннего выявления различных НОС [10]. Функциональная визуализация с помощью позитронно-эмиссионной томогра-

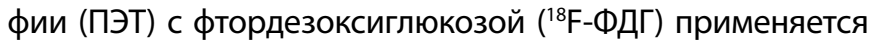
для оценки степени заболевания и для наблюдения после лечения. Альтернативой молекулярной визуализации ПЭТ c ${ }^{18} \mathrm{~F}-$ ФДГ при диагностике опухолей с низким метаболизмом глюкозы (например, рака щитовидной железы) может выступать диффузионная МРТ, которая не требует использования контраста [11]. Компьютерная томография (КT) и ПЭТ/КТ также применяются для онкологической визуализации, однако имеют ограничения для педиатрических пациентов в связи с кумулятивным облучением [12].

Ключевой частью скрининга НОС является генетический анализ. Выявление патогенной мутации у больного дает возможность подтвердить, что наблюдаемое состояние является следствием НОС или рассчитать риск развития заболевания и определить прогноз для родственников пациента, которые могут быть проверены на статус носительства мутации.

Крупные проспективные исследования эффективности существующих программ раннего выявления продемонстрировали преимущества регулярного скрининга людей из групп риска $[13,14]$.

\section{КОНСУЛЬТАЦИЯ ГЕНЕТИКА}

Консультация врача-генетика, специализирующегося в области онкогенетики, является обязательной при подозрении НОС. В противном случае существует вероятность ошибочного выбора методов тестирования и неверной интерпретации полученных данных $[15,16]$.

Вначале врач-генетик описывает индивидуальную и семейную историю для определения вероятности наличия НОС, а также возможные варианты НОС. После этого определяются генетические тесты и другие исследования (лабораторные и инструментальные), необходимые для подтверждения диагноза.

Генетическое тестирование может быть более информативным при наличии прямого родственника с ранее выявленным или текущим диагнозом рака. Если у одного члена семьи будет подтвержден НОС, то все родственники (имеющие или нет опухолевые заболевания или другие симптомы), которые могут унаследовать его, должны рассмотреть возможность генетического тестирования. Знание существующих рисков развития рака позволит выбрать тактику ведения пациентов для предотвращения или раннего выявления заболевания.

После получения результатов генетических тестов в обязательном порядке проводится еще одна консультация врача-генетика для корректной интерпретации выявленных мутаций и формирования рекомендаций по дальнейшему наблюдению пациента. Врач должен разъяснить генетические и клинические аспекты заболевания (имеющегося или потенциального), донести плюсы и минусы генетического тестирования для кровных родственников пациента [17].

Необходимо помнить, что при любом генетическом тестировании существует ряд неопределенностей, затрудняющих индивидуальный прогноз (именно поэтому важно наличие опыта у врача, интерпретирующего данные). Во-первых, не у всех носителей патогенных мутаций разовьется рак. Важным критерием является так называемая пенетрантность выявленных вариантов генов. Во-вторых, существуют различия в экспрессивности, когда люди, наследующие один вариант, могут различаться по степени проявления признаков и симптомов синдрома, включая развитие ассоциированного рака [18]. В частности, на это влияют образ жизни и экологические факторы. В-третьих, результаты тестирования могут оказаться неинформативными, например, у пациента выявлены мутации с неизвестным клиническим значением (variant of uncertain significance, VUS). Некоторые VUS впоследствии могут быть реклассифицированы как ассоциированные с повышенным риском развития рака, поэтому генетической лаборатории важно поддерживать связь с пациентом для информирования о возможных изменениях в интерпретации результатов его теста. В-четвертых, даже отрицательный результат не означает отсутствие НОС, т.к. тестирование могло затрагивать не все патогенные мутации (например, если некоторые из них пока неизвестны) [19]. В последнем случае, когда известные патогенные мутации не выявлены, но семейный анамнез четко указывает на наличие НОС, целесообразно применять меры профилактики и вести наблюдение.

\section{ГЕНЕТИЧЕСКОЕ ТЕСТИРОВАНИЕ}

Для анализа на герминальные мутации может быть использован различный биологический материал, однако чаще всего это кровь пациента, собранная в пробирку с антикоагулянтом (этилендиаминтетраацетатом (ЭДТА), цитратом натрия) или высушенная на фильтре.

Изначально генетическое тестирование при подозрении на НОС проводилось для отдельных генов с использованием метода секвенирования по Сэнгеру. Например, для подтверждения синдрома Линча анализировались гены системы репарации ДНК - MLH1, MSH2 и MSH6 [20]. При использовании метода Сэнгера для минимизации стоимости тестирование проводится последовательно, при этом врачу необходимо определить порядок проверки патогенных вариантов от наиболее вероятных к менее вероятным. 
С постепенным открытием большего числа патогенных мутаций, а также с развитием технологий высокопроизводительного секвенирования (Next Generation Sequencing, NGS) все большее практическое значение приобретает мультигенное тестирование с одновременным анализом множества генов [21]. Это сокращает время до получения результатов, когда необходимо проанализировать три и более генов. Так как тестирование включает анализ генов с разной пенетрантностью, в отдельных случаях даже низкой или неизвестной, при более широком его использовании в клинической практике происходит интенсивное накопление информации о новых патогенных вариантах, а также уточнение пенетрантности уже известных [22]. Кроме того, применение метода NGS позволяет сопоставить определенные сочетания мутаций с наблюдаемым при НОС фенотипом, выявить новые корреляции мутаций и повышенного риска рака (например, повышение вероятности рака молочной железы при наличии мутации в гене PMS2) [23]. В то же время повышается вероятность обнаружения мутаций с неизвестным клиническим значением. Это, а также отсутствие клинических рекомендаций при выявлении вариантов со средней или низкой пенетрантностью затрудняет интерпретацию данных [24].

Еще больше информации и одновременно неопределенности дает использование полноэкзомного и полногеномного секвенирования. Таким способом могут быть получены данные о новых патогенных вариантах, но для определения их пенетрантности требуется исследование больших групп пациентов на протяжении длительного времени [25]. С учетом высокой стоимости анализа такой подход редко применяется в клинической практике, однако потенциально имеет перспективы более широкого использования, в том числе ввиду постепенного снижения цены. Применение полноэкзомного и полногеномного секвенирования целесообразно, если другие генетические методы не позволяют выявить причину НОС [26].

Вне зависимости от того, какой был получен результат, генетическое тестирование может быть полезно в следующих случаях [27]:

- если результат является положительным, пациент будет иметь возможность управлять рисками (например, смена образа жизни, регулярное прохождение обследований или превентивное хирургическое вмешательство могут повысить шансы на благополучный исход);

- если рак уже диагностирован, результаты генетического тестирования могут быть полезны при выборе методов терапии;

- если результат является информативным отрицательным, пациент будет знать, что не унаследовал патогенный вариант гена, что важно для психологического спокойствия;

- при положительном результате члены семьи также будут иметь возможность узнать о своих рисках НОС.

Однако возможны и негативные последствия генетического тестирования [28]. В первую очередь это психологический стресс при обнаружении патогенного варианта или при получении неинформативного результата (например, при обнаружении VUS). При отрицательном результате тестирования возможно чувство вины перед родственниками, у которых были выявлены генетические нарушения. Надо отметить и то, что стоимость генетического тестирования, хоть и постоянно снижается, остается высокой. Поэтому очень важно, чтобы пациент давал информированное согласие на генетическое тестирование, осознавая как его возможную пользу, так и существующие ограничения.

\section{ПРОФИЛАКТИКА И ТЕРАПИЯ НАСЛЕДСТВЕННЫХ ОПУХОЛЕВЫХ СИНДРОМОВ}

После подтверждения НОС к пациентам должны применяться особые подходы для профилактики и терапии заболевания. В случае если заболевание еще не манифестировало, основой профилактики является регулярное прохождение осмотров и определение уровней биомаркеров (таблица). При выявлении некоторых мутаций рекомендованы профилактические операции по удалению органа, подверженного высокому риску развития рака. Например, при отдельных мутациях гена RET пациентам с синдромом множественных эндокринных неоплазий показана тиреоидэктомия в течение первого года жизни [29]. При мутациях в TP53, BRCA1, BRCA2, связанных с повышенным риском развития рака молочной железы, контралатеральная профилактическая мастэктомия [30].

Все больше данных свидетельствует о необходимости использования персонифицированного подхода к выбору специфических методов терапии НОС, к которым относятся:

- таргетная терапия, например, использование ингибиторов тирозинкиназы RET для больных с наследственным раком щитовидной железы, имеющих нарушения в гене $R E T$ [31], и применение ингибиторов PARP для пациентов с раком молочной железы и мутациями BRCA [32];

- решение вопроса об исключении радиотерапии, например, при мутациях ТР53 (т.к. в этом случае риск новых постлучевых новообразований выше, чем риск от наличия регионарных метастазов) [33];

- изменение схем лечения, например, связанных с повышенной чувствительностью опухоли к определенным препаратам (например, к цисплатину у пациентов с мутациями BRCA1) [34].

Таким образом, знание генетического портрета пациента имеет диагностическое, прогностическое и терапевтическое значение.

\section{РАСПРОСТРАНЕННЫЕ НАСЛЕДСТВЕННЫЕ ОПУХОЛЕВЫЕ СИНДРОМЫ}

Наследственный неполипозный колоректальный рак (ННКР, синдром Линча, НNPCC)

Рак ободочной (толстой) кишки является одним из наиболее распространенных онкологических заболеваний.

По данным за 2018 г., в мире диагностировано 1800977 случаев ЗНО толстой кишки, умерли 861663 больных, что составило около 9\% от общего количества смертей от $3 \mathrm{HO}$ [35]. В России зарегистрировано около 72000 новых случаев колоректального рака (КРP) в 2017 г., для 35267 больных заболевание стало причиной смерти. В структуре заболеваемости $3 \mathrm{HO}$ среди пациентов обоих полов рак толстой кишки занимал 2-е место в 2017 г. [36]. Около 3-5\% случаев КРР связано с одним из наследственных синдромов, ассоциированных с раком толстой кишки [37, 38, 39, таблица]. 
Таблица. Международные рекомендации по скринингу и лечению наследственных опухолевых синдромов в зависимости от выявленных генетических изменений (начало)

\begin{tabular}{|c|c|c|c|}
\hline Ген & $\begin{array}{l}\text { Синдром/вовлечение } \\
\text { эндокринной системы }\end{array}$ & Сообщество & $\begin{array}{c}\text { Рекомендованный возраст начала скрининговых } \\
\text { мероприятий/лечения }\end{array}$ \\
\hline$B R C A 1 / B R C A 2$ & $\begin{array}{l}\text { Наследственный рак } \\
\text { молочной железы } \\
\text { и яичников/ } \\
\text { рак поджелудочной железы }\end{array}$ & NCCN, CAPS & $\begin{array}{l}\text { Самообследование молочных желез (18 лет), } \\
\text { обследование у врача-маммолога (25 лет), } \\
\text { МРТ молочных желез (25 лет), маммография } \\
\text { (30 лет), мастэктомия (индивидуально с учетом } \\
\text { клинико-анамнестических данных), СА-125 } \\
\text { и трансвагинальное УзИ (30 лет), удаление яичников } \\
\text { и труб (35-40 лет - BRCA1,35-45 лет - BRCA2), } \\
\text { обследование грудной железы у мужчин (35 лет), } \\
\text { скрининг рака простаты (40 лет) }\end{array}$ \\
\hline
\end{tabular}

\begin{tabular}{|c|c|}
\hline MLH1 & \\
\hline MSH2 & Синдром Линча \\
\hline & (семейный неполипозный \\
\hline MSH6 & рак толстой кишки)/ \\
\hline PMS2 & $\begin{array}{l}\text { яак Поджелудочнои железы, } \\
\text { яичников }\end{array}$ \\
\hline
\end{tabular}

Колоноскопия (20-25 лет), гистерэктомия и сальпингоофорэктомия (после рождения

NCCN, CAPS USMSTF детей), исследования эндометрия, СА-125 и трансвагинальное УЗИ (30-35 лет), уринолизис (25-35 лет), прием аспирина, колоректальные исследования, скрининг, обследования поджелудочной железы

EPCAM

\begin{tabular}{|c|c|c|c|}
\hline$A P C$ & $\begin{array}{l}\text { Семейный аденоматозный } \\
\text { полипоз/ } \\
\text { дифференцированный } \\
\text { рак щитовидной железы } \\
\text { (часто папиллярный); рак } \\
\text { поджелудочной железы }\end{array}$ & NCCN & $\begin{array}{l}\text { Колоноскопия/сигмоидоскопия (10-15 лет), } \\
\text { колоректальные обследования, МРТ/КТ (20-25 лет), } \\
\text { пальпация щитовидной железы + УзИ (поздний } \\
\text { пубертат), пальпация живота, МРТ/КТ по показаниям }\end{array}$ \\
\hline $\begin{array}{l}\text { MUTYH } \\
(2 \text { konuu) }\end{array}$ & $\begin{array}{l}\text { MUTYH-ассоциированный } \\
\text { полипоз/ } \\
\text { рак яичников }\end{array}$ & $\mathrm{NCCN}$ & $\begin{array}{l}\text { Колоноскопия (25-30 лет), колоректальные } \\
\text { исследования, эндоскопия (30-35 лет) }\end{array}$ \\
\hline $\begin{array}{l}\text { MUTYH } \\
\text { (1 копия) }\end{array}$ & $\begin{array}{l}\text { MUTYH-ассоциированный } \\
\text { колоректальный рак/ } \\
\text { рак яичников }\end{array}$ & $\mathrm{NCCN}$ & Колоноскопия (40 лет) \\
\hline $\begin{array}{l}\text { CDKN2A } \\
\text { CDK4 }\end{array}$ & $\begin{array}{l}\text { Меланома/ } \\
\text { рак поджелудочной железы }\end{array}$ & MGC & Защита кожи, обследование у дерматолога (10 лет) \\
\hline PTEN & $\begin{array}{l}\text { Синдром PTEN-гамартом/ } \\
\text { дифференцированный } \\
\text { рак щитовидной железы } \\
\text { (особенно фолликулярный); } \\
\text { многоузловой зоб }\end{array}$ & $\mathrm{NCCN}$ & $\begin{array}{l}\text { Самообследование молочных желез (18 лет), } \\
\text { МРТ молочных желез (30-35 лет), маммография } \\
\text { (30-35 лет), мастэктомия (индивидуально), биопсия } \\
\text { эндометрия/УЗИ (30-35 лет), гистерэктомия, } \\
\text { УзИ щитовидной железы (с момента постановки } \\
\text { диагноза), колоноскопия (35 лет), УзИ почек (40 лет) }\end{array}$ \\
\hline TP53 & $\begin{array}{l}\text { Ли-Фраумени/ } \\
\text { адренокортикальная } \\
\text { карцинома; } \\
\text { дифференцированный рак } \\
\text { щитовидной железы }\end{array}$ & NCCN & $\begin{array}{l}\text { Комплексное клиническое обследование в } \\
\text { детстве, самообследование молочных желез } \\
\text { (18 лет), обследования у маммолога (20-25 лет), } \\
\text { МРТ всего тела (индивидуально), МРТ молочных } \\
\text { желез (20 лет), маммография (30 лет), мастэктомия } \\
\text { (индивидуально), колоноскопия (25 лет), } \\
\text { обследования кожи }\end{array}$ \\
\hline STK11 & $\begin{array}{l}\text { Синдром Пейтца-Егерса/ } \\
\text { рак поджелудочной } \\
\text { железы; стромальные } \\
\text { опухоли яичников; рак } \\
\text { яичек (крупноклеточная } \\
\text { кальцифицирующая опухоль } \\
\text { клеток Сертоли) }\end{array}$ & NCCN, CAPS & $\begin{array}{l}\text { Маммография и МРТ молочных желез (25 лет), } \\
\text { колоноскопия и эзофагогастродуоденоскопия } \\
\text { (поздний пубертат), КТ или МРТ (8-10 лет), } \\
\text { обследование поджелудочной железы и другие } \\
\text { виды скрининга (30-35 лет), УзИ органов малого таза } \\
\text { (18-20 лет), обследования яичек (10 лет) }\end{array}$ \\
\hline
\end{tabular}


Таблица. Международные рекомендации по скринингу и лечению наследственных опухолевых синдромов в зависимости от выявленных генетических изменений (окончание)

\begin{tabular}{|c|c|c|c|}
\hline Ген & $\begin{array}{l}\text { Синдром/вовлечение } \\
\text { эндокринной системы }\end{array}$ & Сообщество & $\begin{array}{c}\text { Рекомендованный возраст начала скрининговых } \\
\text { мероприятий/лечения }\end{array}$ \\
\hline $\mathrm{CDH} 1$ & $\begin{array}{l}\text { Наследственный диффузный } \\
\text { рак желудка/ } \\
\text { карцинома вилочковой } \\
\text { железы; рак яичников }\end{array}$ & $\mathrm{NCCN}$ & $\begin{array}{l}\text { Эндоскопия с биопсией, гастрэктомия (18 лет), } \\
\text { колоноскопия (40 лет), лечение Helicobacter pylori, } \\
\text { самообследование молочных желез (18 лет), } \\
\text { обследование у врача-маммолога (25 лет), МРТ } \\
\text { молочных желез (25 лет); маммография (30 лет), } \\
\text { мастэктомия (индивидуально) }\end{array}$ \\
\hline BMPR1A & $\begin{array}{l}\text { Синдром ювенильного } \\
\text { полипоза/ } \\
\text { рак поджелудочной железы }\end{array}$ & NCCN & $\begin{array}{l}\text { Колоноскопия и эндоскопия верхних отделов } \\
\text { (15 лет), обследования прямой кишки }\end{array}$ \\
\hline SMAD4 & $\begin{array}{l}\text { Синдром ювенильного } \\
\text { полипоза } \\
\text { (Hereditary Hemorrhagic } \\
\text { Telangiectasia (HНT))/ } \\
\text { рак поджелудочной железы }\end{array}$ & NCCN, HHT & $\begin{array}{l}\text { Колоноскопия и эндоскопия верхних отделов } \\
\text { (15 лет), обследования прямой кишки; контроль } \\
\text { анемии; МРТ головного мозга; могут быть } \\
\text { рекомендованы Эхо-КГ и КТ органов грудной } \\
\text { полости }\end{array}$ \\
\hline PALB2 & $\begin{array}{l}\text { PALB2-ассоциированный } \\
\text { риск развития рака/ } \\
\text { рак поджелудочной железы }\end{array}$ & NCCN & $\begin{array}{l}\text { Маммография и МРТ молочных желез (30 лет); } \\
\text { обследование поджелудочной железы; возможна } \\
\text { мастэктомия; скрининг рака грудной железы } \\
\text { у мужчин }\end{array}$ \\
\hline CHEK2 & $\begin{array}{l}\text { СНЕК2-ассоциированный } \\
\text { риск развития рака/ } \\
\text { рак щитовидной железы }\end{array}$ & NCCN & $\begin{array}{l}\text { Маммография и МРТ молочных желез (40 лет); } \\
\text { мастэктомия с учетом семейного анамнеза; } \\
\text { колоноскопия ( } 40 \text { лет); скрининг рака грудной } \\
\text { железы у мужчин }\end{array}$ \\
\hline ATM & $\begin{array}{l}\text { ATM-ассоциированный риск } \\
\text { развития рака/ } \\
\text { рак поджелудочной железы }\end{array}$ & NCCN, CAPS & $\begin{array}{l}\text { Маммография и МРТ молочных желез (40 лет); } \\
\text { обследование поджелудочной железы; др. виды } \\
\text { скрининга }\end{array}$ \\
\hline$N B N$ & $\begin{array}{l}N B N \text {-ассоциированный риск } \\
\text { развития рака/ } \\
\text { рак яичников }\end{array}$ & NCCN, AUA & $\begin{array}{l}\text { Скрининг рака простаты (45 лет); маммография } \\
\text { и МРТ молочных желез (40 лет) }\end{array}$ \\
\hline BARD1 & $\begin{array}{l}\text { BARD1-ассоциированный } \\
\text { риск развития рака }\end{array}$ & & $\begin{array}{l}\text { Индивидуальный план скрининга рака молочной } \\
\text { железы }\end{array}$ \\
\hline BRIP1 & $\begin{array}{l}\text { BRIP1-ассоциированный } \\
\text { риск развития рака/ } \\
\text { рак яичников }\end{array}$ & NCCN & $\begin{array}{l}\text { Двусторонняя сальпингоовариэктомия; } \\
\text { индивидуальный скрининг рака молочной железы } \\
\text { и яичников, мероприятия по снижению риска }\end{array}$ \\
\hline RAD51C & $\begin{array}{l}\text { RAD51C-ассоциированный } \\
\text { риск развития рака/ } \\
\text { рак яичников }\end{array}$ & $\mathrm{NCCN}$ & $\begin{array}{l}\text { Двусторонняя сальпингоовариэктомия; } \\
\text { индивидуальный скрининг рака молочной железы } \\
\text { и яичников, мероприятия по снижению риска }\end{array}$ \\
\hline RAD51D & $\begin{array}{l}\text { RAD51D-ассоциированный } \\
\text { риск развития рака/ } \\
\text { рак яичников }\end{array}$ & NCCN & $\begin{array}{l}\text { Двусторонняя сальпингоовариэктомия; } \\
\text { индивидуальный скрининг рака молочной железы } \\
\text { и яичников, мероприятия по снижению риска }\end{array}$ \\
\hline POLD1 & $\begin{array}{l}\text { Предрасположенность } \\
\text { к колоректальному раку } \\
\text { (Polymerase proofreading- } \\
\text { associated syndrome/ } \\
\text { polyposis) }\end{array}$ & NCCN & $\begin{array}{l}\text { Колоноскопия (25-30 лет), инструментальное } \\
\text { обследование толстой и прямой кишки }\end{array}$ \\
\hline
\end{tabular}

Предрасположенность к колоректальному раку POLE
(Polymerase proofreading- NCCN associated syndrome/ polyposis)
Колоноскопия (25-30 лет), инструментальное обследование толстой и прямой кишки

NCCN - National Comprehensive Cancer Network; CAPS - Cancer of the Pancreas Screening; AUA - American Urological Association; HHT HHT Foundation International — Guidelines Working Group; USMSTF — US Multisociety Task Force on Colorectal Cancer. 
ННКР является наиболее частой причиной наследственного колоректального рака. Этот синдром вызван мутациями в генах репарации неспаренных оснований ДНК. Характерным признаком аутосомно-доминантного наследственного синдрома является раннее возникновение преимущественно правосторонних колоректальных карцином, часто синхронных и метахронных злокачественных образований в эндометрии, почечной лоханке, мочевыводящих путях, тонкой кишке, желудке, яичниках, поджелудочной железе, желчных протоках, головном мозге и коже. Степень пенетрантности для этого заболевания составляет 80-90\% [40, 41]. Примерно каждый 500-й человек в общей популяции является носителем мутаций, характерных для ННКР [42].

Клинический диагноз ННКР ставится при соблюдении критериев Amsterdam II [41]. Поскольку многие семьи не могут строго соответствовать этим критериям ввиду небольшого количества родственников и неполной пенетрантности, дополнительно установлены следующие критерии Bethesda.

Диагностические критерии для ННКР (требуется соблюдение всех) ${ }^{*}$ :

1. три и более случаев гистологически подтвержденных ЗНО в семье: КРР, рак эндометрия, тонкой кишки, мочеточника или почечной лоханки; один из членов семьи является родственником I степени родства относительно двух других; должен быть исключен семейный аденоматозный полипоз;

2. в двух и более последовательных поколениях встречается 3НO;

3. диагноз в возрасте до 50 лет по крайней мере у одного члена семьи.

Соответствие этим критериям может указывать на наличие ННКР, но не является окончательным диагнозом. Дальнейшая генетическая диагностическая оценка для ННКР должна быть выполнена в следующих случаях (критерии Bethesda $[42,43])$ :

1. пациенты с раком толстой кишки в возрасте до 50 лет;

2. пациенты с двумя или более опухолями в анамнезе, ассоциированными с ННКР;

3. пациенты с раком толстой кишки, имеющие по крайней мере одного родственника I степени с опухолью, ассоциированной с ННКР, в возрасте до 50 лет;

4. пациенты с раком толстой кишки, имеющие как минимум двух родственников с опухолью, ассоциированной с НHКР;

5. пациенты с колоректальными аденомами в возрасте до 40 лет без признаков полипозной болезни;

6. лица с родственником I степени, который удовлетворяет одному из первых пяти критериев.

Для новообразований, связанных с синдромом Линча, характерна микросателлитная нестабильность (накопление клетками большого количества мутаций вследствие нарушения системы репарации ДНК), что может быть определено с помощью тестирования ДНК.

Семейный аденоматозный полипоз (САП, FAP) обычно характеризуется развитием от сотен до тысяч аденоматозных полипов по всей ободочной кишке. Рост аденом, как правило, начинается на втором десятилетии жизни в ректосигмоидальном отделе. При отсутствии

* на основе критериев Amsterdam II [41]. лечения пациенты умирают от рака в среднем в возрасте 40 лет.

Помимо классического САП, существует также более легкое течение заболевания - аттенуированная форма, при которой у пациентов обычно развивается менее 100 аденом. Манифестация заболевания, как правило, на 10-15 лет позже, чем при классическом САП. Аденомы часто локализуются в проксимальном отделе толстой кишки. У некоторых пациентов также выявляются опухоли за пределами кишечника, особенно аденомы дна желудка, остеомы нижней и верхней челюстей, длинных трубчатых костей, рак щитовидной и поджелудочной желез, а также десмоидные опухоли (примерно в 13\% случаев). Патогномоничным признаком для этого заболевания является характерная врожденная гипертрофия пигментного эпителия сетчатки (congenital hypertrophy of the retinal pigment - CHRPE), которая присутствует примерно у 85\% пациентов, но не влияет на зрение.

Риск смерти пациентов с САП от КРР может быть уменьшен с помощью усиленной программы раннего выявления и профилактической колэктомии.

Дифференциальная диагностика при САП:

- классический семейный аденоматозный полипоз (САП, FAР);

- аттенуированная форма семейного аденоматозного полипоза (АСАП, АFAP);

- МUTYH-ассоциированный полипоз.

Частота заболевания в общей популяции составляет 1:33 000, пенетрантность близка к 100\%. САП вызывается герминальной мутацией в гене-супрессоре опухоли APC. Лица, подверженные риску САП, должны ежегодно проходить ректосигмоидоскопию с 10 лет. Решение о необходимости операции принимается на основании клинических данных. После сохраняющей прямую кишку колэктомии риск развития карциномы культи прямой кишки составляет около 13\% в течение 25 лет после оперативного лечения [44]. В нескольких исследованиях было продемонстрировано, что вероятность смерти пациентов с САП от КРР была заметно снижена благодаря программе регулярного скрининга и профилактической колэктомии [37].

MUTYH-ассочиированные опухоли клинически сопоставимы с аттенуированной формой САП. Заболевание вызвано герминальными мутациями и является одним из немногих НОC с аутосомно-рецессивным типом наследования. Эти пациенты, соответственно, получили материнскую и отцовскую поврежденные копии гена MUTYH. Наличие MUTYH-ассоциированного рака следует заподозрить, если рак диагностируется в молодом возрасте у отдельных пациентов или у братьев и сестер, чьи родители здоровы, и/или при наличии от 15 до 20 аденом толстой кишки. Описаны неколоректальные проявления в виде рака яичников [45].

В России у всех пациентов с гистологически подтвержденным КРP рекомендовано проанализировать семейный анамнез. При диагностированном метастатическом процессе или подозрении на него рекомендовано выполнять анализ опухолевой ткани на мутации экзонов 2-4 генов KRAS и NRAS, поскольку выявленные генетические особенности могут влиять на тактику лечения (выбор таргетного агента) при наличии отдаленных метастазов. 
Проведение генетического тестирования рекомендовано при подозрении на ННКР (анализ мутаций в генах MLH1, MSH2, MSH6, PMS2); САП (анализ мутаций в гене APC); АСАП и MUTYH-ассоциированный полипоз (анализ мутаций в гене $M U T Y H)$.

Большинство НОС, связанных с развитием КРР, наследуются по аутосомно-доминантному типу. Исключениями являются MUTYH-ассоциированный полипоз и синдром Блума. Если САП и ННКР не подтверждены, показано медико-генетическое консультирование с целью выявления более редких синдромов: Пейтца-Егерса, ЛиФраумени, Коудена, Блума и других [46, таблица].

Наследственный рак молочной железы и яичников (HBOC syndrome)

Приблизительно у каждой двенадцатой женщины развивается рак молочной железы в течение жизни.

Основными этиопатогенетическими факторами наследственного рака молочной железы и яичников (РМЖ и РЯ) являются изменения в генах BRCA1 и BRCA2. Около 5\% случаев РМЖ обусловлены мутацией в одном из двух генов [47]. Известны и другие гены, мутации в которых ассоциированы с наследственными формами РМЖ и РЯ [48, таблица]. У носителей мутаций в генах BRCA1 или BRCA2 риск развития РМЖ и РЯ в течение жизни составляет в среднем 80\% и 20-40\% соответственно [49, 50]. Мужчины-носители мутации обычно не заболевают, но имеют повышенный риск рака грудной железы и могут передавать мутацию своему потомству. У мужчин-носителей мутации BRCA1 в большей степени повышен риск рака простаты, с мутацией BRCA2 высока вероятность рака грудной железы.

Выявление наиболее частых наследственных дефектов в генах BRCA1 и BRCA2 стало рутинным компонентом обследования пациенток с карциномами молочной железы и яичника. Целью подобных мероприятий является не только поиск условно здоровых родственниц-носительниц мутаций $B R C A 1 / 2$, но и модификация схемы лекарственного лечения уже имеющегося онкологического заболевания.

Несмотря на интенсивную динамику формирования и изменения критериев для поиска мутаций в генах BRCA1 и BRCA2 в международной и российской практике, можно выделить основные из них:

1. РМЖ выявлен в возрасте до 50 лет;

2. билатеральный РМЖ;

3. РМЖ и РЯ у того же самого пациента или в одной семье;

4. два или более типов рака, которые могут быть связаны с мутациями BRCA1/2, у одного и того же члена семьи;

5. случаи РМЖ у мужчин;

6. этническая принадлежность к евреям Ашкенази.

Патогенная мутация в генах BRCA1/BRCA2 может быть обнаружена примерно в 50\% семей с подозрением на наследственные формы РМЖ и РЯ. Если наличие в семье РМЖ и/или РЯ сопровождается выявлением мутации, родственникам из группы риска рекомендуется регулярно проходить скрининг для раннего диагностирования заболевания. Следует учитывать, что отрицательный результат тестирования на частые мутации не исключает наличия патогенных изменений в генах $B R C A 1 / 2$ или мутаций других генов, ассоциированных с повышенным риском развития опухолей. Программа раннего выявления лиц с повышенным риском развития наследственного РМЖ и РЯ:

1. регулярное самообследование, пальпаторное обследование молочной железы у маммолога раз в 6 месяцев;

2. УЗИ молочных желез раз в 6 месяцев;

3. МРТ молочных желез раз в год.

Множественная эндокринная неоплазия типа 2 (МЭН2, MEN2)

МЭН2 встречается в субформах МЭН2а и МЭН2b [51]. Характерной чертой МЭН2а является развитие почти в 100\% случаев медуллярного рака щитовидной железы (С-клеточный рак), феохромоцитомы (40-60\%) и гиперпаратиреоза, обусловленного аденомой, гиперплазией или раком околощитовидных желез, которые отмечаются у 20-60\% больных.

При типе МЭН2b медуллярный рак щитовидной железы может встречаться уже в раннем детстве. Пациенты с МЭН2 обычно имеют марфаноподобный тип телосложения, а также грубые черты удлиненного лица и выпуклые губы.

Мутации в протоонкогене RET ответственны за все субформы. Медуллярный рак щитовидной железы относительно редко встречается в популяции. Поскольку от 25 до 33\% случаев заболевания развивается в рамках МЭН2, установленный диагноз медуллярного рака щитовидной железы уже, по сути, является показанием к проведению молекулярно-генетического анализа для МЭН2 [52].

С учетом высокого риска развития $3 \mathrm{HO}$ щитовидной железы у носителей некоторых мутаций нередко рекомендуется профилактическая тиреоидэктомия [53]. Операция может быть рекомендована уже на первом году жизни из-за очень раннего возраста начала заболевания и практически 100\% пенетрантности [54]. Для других сопутствующих заболеваний следует проводить регулярный клинический скрининг.

\section{Синдром Ли-Фраумени}

Для синдрома Ли-Фраумени является характерным высокий риск развития злокачественных опухолей в детском и молодом возрасте: РМЖ, саркомы, опухоли головного мозга, лейкемии, рак почек и надпочечников. Также он связан с повышенным риском развития опухолей других локализаций, включая меланому, рак толстой кишки и рак поджелудочной железы.

Причиной синдрома Ли-Фраумени являются герминальные мутации в гене-супрессоре опухоли ТР53. Белок p53 играет ключевую роль в регуляции клеточного цикла. Риск развития рака для носителей мутации ТР53 в течение жизни составляет около 85\% [55]. Для них характерно развитие первично-множественных опухолей в разных органах. Поскольку спектр опухолей очень широк, в настоящее время не существует целевой программы раннего выявления этого синдрома. Наличие мутаций в ТР53 может приводить к развитию РМЖ в возрасте до 30 лет [56], а медианный возраст составляет 34 года. В мировой практике у женщин есть возможность профилактической мастэктомии для уменьшения риска развития РМЖ [57]. 
В таблице дополнительно представлены гены, анализ мутаций которых рекомендован при подозрении на наследственные формы рака. Для некоторых генетических изменений разработаны международные рекомендации по медицинскому сопровождению пациентов-носителей. Перечень НОС все время расширяется, появляются новые и уточненные данные о различных HOC, однако не вся информация находит отражение В клинических рекомендациях. Актуальный перечень HOC с основными характеристиками их клинических проявлений и генетической основы приведен в базе данных GeneReviews [58].

\section{ЗАКЛЮЧЕНИЕ}

Несмотря на накопленный международный научно-практический опыт в вопросах изучения этиологии и патогенеза наследственных опухолевых синдромов, на территории Российской Федерации имеется необходимость выработки единой тактики медико-генетического консультирования и молекулярно-генетической диагностики для их выявления. Очевидна потребность в формировании клинических рекомендаций по медицинскому сопровождению носителей мутаций в генах, ассоциированных с высоким риском развития наследственных форм рака, и внедрении программы современной молекулярно-генетической диагностики и экспертного медико-генетического консультирования в ведущие медицинские центры страны, специализирующиеся как на отдельных направлениях (например, эндокринология, колопроктология), так и на онкологии в целом.

\section{ДОПОЛНИТЕЛЬНАЯ ИНФОРМАЦИЯ}

Источник финансирования. Поисково-аналитическая работа и подготовка статьи проведены на личные средства авторского коллектива.

Конфликт интересов. Авторы декларируют отсутствие явных и потенциальных конфликтов интересов, связанных с публикацией настоящей статьи.

Участие авторов. Все авторы внесли значимый вклад в написание статьи, прочли и одобрили финальный вариант рукописи.

\section{СПИСОК ЛИТЕРАТУРЫ | REFERENCES}

1. Garber J, Offit K. Hereditary cancer predisposition syndromes. J Clin Oncol. 2005;23(2):276-292. doi: 10.1200/jco.2005.10.042.

2. Harper P. Practical genetic counselling. 6th ed. London: Hodder Arnold; 2004. 409 p.

3. Knudson A. Two genetic hits (more or less) to cancer. Nat Rev Cancer. 2001;1(2):157-162. doi: 10.1038/35101031.

4. Имянитов Е.Н. Общие представления о наследственных опухолевых синдромах // Практическая онкология. - 2014. T.15. - №3. - C. 101-106. [Imianitov EN. Obshchie predstavleniia o nasledstvennykh opukholevykh sindromakh. Prakticheskaia onkologiia. 2014;15(3):101-106. (In Russ.)].

5. Hampel H, Bennett R, Buchanan A, et al. A practice guideline from the American College of Medical Genetics and Genomics and the National Society of Genetic Counselors: referral indications for cancer predisposition assessment. Genet Med. 2014;17(1):70-87. doi: 10.1038/gim.2014.147.

6. Tiwari R, Singh A, Somwaru A, et al. Radiologist's Primer on imaging of common hereditary cancer syndromes. Radiographics. 2019;39(3):759-778. doi: 10.1148/rg.2019180171.

7. Shinagare A, Giardino A, Jagannathan J, et al. Hereditary cancer syndromes: a radiologist's perspective. Am J Roentgenol. 2011;197(6):W1001-W1007. doi: 10.2214/ajr.11.6465

8. Ballinger M, Ferris N, Moodie K, et al. Surveillance in Germline TP53 mutation carriers utilizing whole-body magnetic resonance imaging. JAMA Oncol. 2017;3(12):1735-1736. doi: 10.1001/jamaoncol.2017.

9. Anupindi S, Bedoya M, Lindell R, et al. Diagnostic performance of whole-body MRI as a tool for cancer screening in children with genetic cancer-predisposing conditions. Am J Roentgenol. 2015;205(2):400-408. doi: 10.2214/ajr.14.13663

10. ClinicalTrials.gov [Internet]. Screening with whole body MRI for detection of primary tumors in children and adults with Li-Fraumeni syndrome (LFS) and other cancer predisposition syndromes. Available from: https://www.clinicaltrials.gov/ct2/show/NCT02950987.

11. Kwee T, Takahara T, Ochiai R, et al. Complementary roles of wholebody diffusion-weighted MRI and 18F-FDG PET: the state of the art and potential applications. J Nucl Med. 2010;51(10):1549-1558. doi: 10.2967/jnumed.109.073908.

12. Linet M, Slovis T, Miller D, et al. Cancer risks associated with external radiation from diagnostic imaging procedures. CA Cancer J Clin. 2012;62(2):75-100. doi: 10.3322/caac.21132

13. Aretz S, Vasen H, Olschwang S. Clinical Utility Gene Card for: Familial adenomatous polyposis (FAP) and attenuated FAP
(AFAP) - update 2014. Eur J Hum Genet. 2014;23(6):889-889. doi: 10.1038/ejhg.2014.193

14. Boland $P$, Yurgelun $M$, Boland C. Recent progress in Lynch syndrome and other familial colorectal cancer syndromes. CA Cancer J Clin. 2018:68(3):217-231. doi: 10.3322/caac.21448.

15. Brierley K, Blouch E, Cogswell W, et al. Adverse events in cancer genetic testing. Cancer J. 2012;18(4):303-309. doi: 10.1097/ppo.0b013e3182609490

16. Farmer $\mathrm{M}$, Bonadies $\mathrm{D}$, Mahon $\mathrm{S}$, et al. Adverse events in genetic testing: the fourth case series. Cancer J. 2019;25(4):231-236. doi: 10.1097/ppo.0000000000000391.

17. Daly M, Pilarski R, Yurgelun M, et al. NCCN guidelines insights: genetic/familial high-risk assessment: breast, ovarian, and pancreatic, version 1.2020. J Nat/ Compr Canc Netw. 2020;18(4):380-391. doi: 10.6004/jncen.2020.0017

18. Taeubner J, Wieczorek D, Yasin L, et al. Penetrance and expressivity in inherited cancer Predisposing Syndromes. Trends Cancer. 2018;4(11):718-728. doi: 10.1016/j.trecan.2018.09.002

19. Powers J, Ebrahimzadeh J, Katona B. Genetic testing for hereditary gastrointestinal cancer syndromes: Interpreting results in today's practice. Curr Treat Options Gastroenterol. 2019;17(4):636-649. doi: 10.1007/s11938-019-00253-2.

20. Vasen H, Blanco I, Aktan-Collan K, et al. Revised guidelines for the clinical management of Lynch syndrome (HNPCC): recommendations by a group of European experts. Gut. 2013;62(6):812-823. doi: 10.1136/gutjnl-2012-304356

21. Rahman N. Mainstreaming genetic testing of cancer predisposition genes. Clin Med (Lond). 2014;14(4):436-439. doi: 10.7861/clinmedicine.14-4-436.

22. Susswein L, Marshall M, Nusbaum R, et al. Pathogenic and likely pathogenic variant prevalence among the first 10,000 patients referred for next-generation cancer panel testing. Genet Med. 2015;18(8):823-832. doi: 10.1038/gim.2015.166.

23. Ten Broeke S, Brohet R, Tops C, et al. Lynch Syndrome caused by germline PMS2 mutations: delineating the cancer risk. J Clin Oncol. 2015;33(4):319-325. doi: 10.1200/jco.2014.57.8088.

24. Colas C, Golmard L, de Pauw A, et al. «Decoding hereditary breast cancer» benefits and questions from multigene panel testing. Breast 2019;45:29-35. doi: 10.1016/j.breast.2019.01.002.

25. Shahi R, de Brakeleer S, Caljon B, et al. Identification of candidate cancer predisposing variants by performing whole-exome sequencing on index patients from BRCA1 and BRCA2negative breast cancer families. BMC Cancer. 2019;19(1):313. doi: 10.1186/s12885-019-5494-7 
26. Sponziello M, Benvenuti S, Gentile A, et al. Whole exome sequencing identifies a germline MET mutation in two siblings with hereditary wild-type RET medullary thyroid cancer. Hum Mutat. 2017;39(3):371-377. doi: 10.1002/humu.23378.

27. Manahan E, Kuerer $\mathrm{H}$, Sebastian $\mathrm{M}$, et al. Consensus quidelines on genetic 'testing for hereditary breast cancer from the American society of breast surgeons. Ann Surg Oncol. 2019;26(10):3025-3031. doi: 10.1245/s10434-019-07549-8

28. Lumish $\mathrm{H}$, Steinfeld $\mathrm{H}$, Koval $\mathrm{C}$, et al. Impact of panel gene testing for hereditary breast and ovarian cancer on patients. J Genet Couns. 2017;26(5):1116-1129. doi: 10.1007/s10897-017-0090-y.

29. Lairmore T, Diesen D, Goldfarb M, et al. American Association of Clinical Endocrinologists and American College of Endocrinology Disease State Clinical Review: timing of multiple endocrine neoplasia thyroidectomy and extent of central neck lymphadenectomy. Endocr Pract. 2015;21(7):839-847. doi: 10.4158/ep14463.dscr.

30. Song C, Teo S, Taib N, Yip C. Surgery for BRCA, TP53 and PALB2: a literature review. Ecancermedicalscience. 2018;12:863. doi: 10.3332/ecancer.2018.863.

31. Roskoski R, Sadeghi-Nejad A. Role of RET protein-tyrosine kinase inhibitors in the treatment RET-driven thyroid and lung cancers. Pharmacol Res. 2018;128:1-17. doi: 10.1016/j.phrs.2017.12.021.

32. Lima Z, Ghadamzadeh M, Arashloo F, et al. Recent advances of therapeutic targets based on the molecular signature in breast cancer: genetic mutations and implications for current treatment paradigms. J Hematol Oncol. 2019;12(1):1-25. doi: 10.1186/s13045-019-0725-6.

33. Matsui Y, Tsuchida Y, Keng P. Effects of p53 mutations on cellular sensitivity to ionizing radiation. Am J Clin Oncol. 2001;24(5):486-490. doi: 10.1097/00000421-200110000-00014

34. Gronwald J, Byrski T, Lubinski J, Narod S. Cisplatin in breast cancer treatment in BRCA1 carriers. Hered Cancer Clin Pract. 2012;10 (Suppl. 4):17. doi: 10.1186/1897-4287-10-s4-a17.

35. Bray F, Ferlay J, Soerjomataram I, et al. Global cancer statistics 2018 : GLOBOCAN estimates of incidence and mortality worldwide for 36 cancers in 185 countries. CA Cancer J Clin. 2018;68(6):394-424 doi: 10.3322/caac.21492

36. Злокачественные новообразования в России в 2017 году (заболеваемость и смертность) / Под ред. А.Д. Каприна, В.В. Старинского, Г.В. Петровой. - М.: МНИОИ им. П.А. Герцена - филиал ФГБУ «НМИЦ радиологии» Минздрава России, 2018. - 250 с. [Zlokachestvennye novoobrazovanija $\vee$ Rossii v 2017 godu (zabolevaemost' i smertnost'). Ed. by A.D. Kaprin, V.V. Starinskii, G.V. Petrova. Moscow: MNIOI im. P.A. Gercena - filial FGBU «NMIC radiologii» Minzdrava Rossii; 2018. 250 p. (In Russ).]

37. Wells K, Wise P. Hereditary colorectal cancer syndromes. Surg Clin North Am. 2017;97(3):605-625. doi: 10.1016/j.suc.2017.01.009.

38. Kastrinos F, Syngal S. Inherited colorectal cancer syndromes. Cancer J. 2011;17(6):405-415. doi: 10.1097/ppo.0b013e318237e408.

39. Ma H, Brosens L, Offerhaus $\mathrm{G}$, et al. Pathology and genetics of hereditary colorectal cancer. Pathology. 2018;50(1):49-59. doi: 10.1016/j.pathol.2017.09.004

40. Lynch $\mathrm{H}$, de la Chapelle A. Hereditary colorectal cancer. N Engl J Med. 2003;348(10):919-932. doi: 10.1056/nejmra012242.

41. Vasen $\mathrm{H}$, Watson P, Meclin J, Lynch $\mathrm{H}$. New clinical criteria for hereditary nonpolyposis colorectal cancer (HNPCC, Lynch syndrome) proposed by the International Collaborative Group on HNPCC. Gastroenterology. 1999;116(6):1453-1456. doi: 10.1016/s0016-5085(99)70510-x.

42. Umar A, Boland C, Terdiman J, et al. Revised bethesda guidelines for hereditary nonpolyposis colorectal cancer (Lynch Syndrome) and microsatellite instability. JNCI. 2004;96(4):261-268. doi: 10.1093/jnci/djh034

43. Rodriguez-Bigas M, Boland C, Hamilton S, et al. A national cancer institute workshop on hereditary nonpolyposis colorectal cancer syndrome: meeting highlights and bethesda guidelines. JNCI 1997;89(23):1758-1762. doi: 10.1093/jnci/89.23.1758.

44. Winawer S, Zauber A, Ho M, et al. Prevention of colorectal cancer by colonoscopic polypectomy. N Engl J Med. 1993:329(27):1977-1981. doi: 10.1056/nejm199312303292701.

45. Aretz S, Uhlhaas S, Goergens H, et al. MUTYH-associated polyposis: 70 of 71 patients with biallelic mutations present with an attenuated or atypical phenotype. Int J Cancer. 2006;119(4):807-814. doi: 10.1002/ijc.21905.

46. НМИЦ онкологии им. Н.Н. Петрова [интернет]. Генетическое консультирование при колоректальном раке. [NMITS onkologii im. N.N. Petrova [Internet]. Geneticheskoye konsul'tirovaniye pri kolorektal'nom rake. (In Russ).] Доступно по: https://www.niioncologii.ru/news/geneticheskoe_konsultirovanie_ pri_kolorektalnom_rake. Ссылка активна на 15.05.2020.

47. Anis $\mathrm{AH}$. Mutations and cancer genesis highlights on BRCA and BRCA2 Genes. J Cancer Prev Curr Res. 2015;2(6):00063. doi: 10.15406/jcpcr.2015.02.00063.

48. Apostolou P, Fostira F. Hereditary Breast cancer: the era of new susceptibility genes. Biomed Res Int. 2013;2013:1-11. doi: 10.1155/2013/747318

49. Antoniou A, Pharoah P, McMullan G, et al. Evidence for further breast cancer susceptibility genes in addition to BRCA1 and BRCA2 in a population-based study. Genet Epidemiol. 2001;21(1):1-18. doi: 10.1002/gepi.1014.

50. Walsh T, Casadei $\mathrm{S}$, Coats $\mathrm{K}$, et al. Spectrum of mutations in BRCA1, BRCA2, CHEK2, and TP53 in families at high risk of breast cancer JAMA. 2006;295(12):1379-1388. doi: 10.1001/jama.295.12.1379.

51. Marini F, Falchetti A, del Monte F, et al. Multiple endocrine neoplasia type 2. Orphanet J Rare Dis. 2006;1:45 doi: 10.1186/1750-1172-1-45.

52. Аметов А.С., Дэпюн Т.И., Позднякова Н.В., и др. Генетические маркеры в диагностике рака щитовидной железы // Эндокринология: новости, мнения, обучение. - 2018. - Т.7. №1. - C.42-49. [Ametov AS, Depui TI, Pozdnyakova NV, et al. Genetic markers in the diagnosis of thyroid cancer. Endocrinology: news, opinions, training. 2018;7(1):42-49. (In Russ.)].

53. Поляков В.Г., Шишков Р.В., Ильин А.А., и др. Результать профилактической тиреоидэктомии у носителей герминальной мутации в гене RET в семьях с наследственными формами медуллярного рака щитовидной железы // Клиническая и экспериментальная тиреоидология. — 2016. - Т.12. — №1. C. 22-33. [Polyakov VG, Shishkov RV, Ilyin AA, et al. Prophylactic thyroidectomy results among RET germline mutation bearers in families with hereditary forms of medullary thyroid cancer. Clinical and experimental thyroidology. 2016;12(1):22-33. (In Russ.)]. doi: 10.14341/ket2016122-33.

54. Романчишен А.Ф., Решетов И.В., Матвеева 3.С., Вабалайте К.В. Семейные формы рака щитовидной железы // Голова и шея. 2015. - №3. - C. 43-46. [Romanchishen AF, Reshetov IV, Matveeva ZS, Vabalaite KV. Family forms of thyroid cancer. Head and neck. 2015:(3):43-46. (In Russ.)].

55. Le Bihan C, Moutou C, Brugières L, et al. ARCAD: A method for estimating age-dependent disease risk associated with mutation carrier status from family data. Genet Epidemiol. 1995;12(1):13-25. doi: 10.1002/gepi.1370120103.

56. McCuaig J, Armel S, Novokmet A, et al. Routine TP53 testing for breast cancer under age 30: ready for prime time? Fam Cancer. 2012;11(4):607-613. doi: 10.1007/s10689-012-9557-z.

57. Thull D, Vogel V. Recognition and management of hereditary breast cancer syndromes. Oncologist. 2004;9(1):13-24. doi: 10.1634/theoncologist.9-1-13.

58. Adam MP, Ardinger HH, Pagon RA, Wallace SE. Gene Reviews [Internet]. Seattle (WA): University of Washington, Seattle; 1993-2020. Available from: https://www.ncbi.nlm.nih.gov/books/NBK1116/.

Рукопись получена: 23.03.2020. Одобрена к публикации: 03.08.2020. Опубликована online: 24.09.2020. 


\section{ИНФОРМАЦИЯ ОБ АВТОРАХ [AUTHORS INFO]}

*Баранова Елена Евгеньевна, к.м.н. [Elena E. Baranova, MD, PhD]; адрес: г. Москва, ул. Баррикадная, д. 2/1, стр. 1, 123242 [address: Barrikadnaya ul., 2/1, str. 1, Moscow, 123242]; ORCID: https://orcid.org/0000-0001-9638-2303; eLibrary SPIN: 3163-6666; e-mail: baranova.gen@gmail.com

Бодунова Наталья Александровна, к.м.н. [Natalia A. Bodunova, MD, PhD]; e-mail: n.bodunova@mknc.ru; ORCID: https://orcid.org/0000-0002-3119-7673; eLibrary SPIN: 3341-2523.

Воронцова Мария Владимировна, к.м.н. [Maria V. Vorontsova, MD, PhD]; e-mail: maria.v.vorontsova@mail.ru; ORCID: https://orcid.org/0000-0002-9124-294X; eLibrary SPIN: 4168-6851.

Захарова Галина Сергеевна, к.Х.H. [Galina S. Zakharova, PhD]; e-mail: galina.s.zakharova@gmail.com; ORCID: https://orcid.org/0000-0002-2548-8511; eLibrary SPIN: 3774-6551.

Макарова Мария Владимировна [Maria V. Makarova, MD]; e-mail: makarova@evogenlab.ru; ORCID: https://orcid.org/0000-0003-1581-9118; eLibrary SPIN: 1638-2012.

Румянцев Павел Олегович, д.м.н. [Pavel O. Rumyantsev, MD, PhD]; e-mail: pavelrum@gmail.com; ORCID: https://orcid.org/0000-0002-7721-634X; eLibrary SPIN: 7085-7976.

Хатьков Игорь Евгеньевич, д.м.н., профессор [Igor E. Hat'kov, MD, PhD, Professor]; e-mail: i.hatkov@mknc.ru; ORCID: https://orcid.org/0000-0002-4088-8118; eLibrary SPIN: 5128-5820.

\section{ЦИТИРОВАТЬ:}

Баранова Е.Е., Бодунова Н.А., Воронцова М.В., Захарова Г.С., Макарова М.В., Румянцев П.О., Хатьков И.Е. Наследственные опухолевые синдромы: современная парадигма // Проблемы эндокринологии. — 2020. — Т. 66. — №4. — С. $24-34$. doi: https://doi.org/10.14341/probl12366

\section{TO CITE THIS ARTICLE:}

Baranova EE, Bodunova NA, Vorontsova MV, Zakharova GS, Makarova MV, Rumyantsev PO, Hat'kov IE. Hereditary cancer syndromes: a modern paradigm. Problems of Endocrinology. 2020;66(4):24-34. doi: https://doi.org/10.14341/probl12366 